\title{
Erratum to: Extremal indices, geometric ergodicity of Markov chains, and MCMC
}

\author{
Gareth O. Roberts · Jeffrey S. Rosenthal • \\ Johan Segers • Bruno Sousa
}

Published online: 4 February 2010

(C) Springer Science+Business Media, LLC 2010

\section{Erratum to: Extremes \\ DOI 10.1007/s10687-006-0028-5}

In the beginning of the proof of Theorem 4.1, the first inequality in the first display is invalid. Here is the correct argument:

$$
\begin{aligned}
\operatorname{Pr}\left(X_{k}>u \mid X_{0}>u\right) & =\int_{u}^{x_{+}} P^{k}(x,(u, \infty)) \frac{\pi(\mathrm{d} x)}{\pi(u, \infty)} \\
& \stackrel{\text { (A) }}{\leq} \int_{u}^{x_{+}} \int_{u}^{x_{+}} \frac{V(y)}{V(u)} P^{k}(x, \mathrm{~d} y) \frac{\pi(\mathrm{d} x)}{\pi(u, \infty)}
\end{aligned}
$$

The online version of the original article can be found at http://dx.doi.org/10.1007/s10687-006-0028-5.

G. O. Roberts ( $ه)$

Department of Mathematics and Statistics, Fylde College, Lancaster University,

LA1 4YF Lancaster, England

e-mail: Gareth.O.Roberts@warwick.ac.uk

\section{J. S. Rosenthal}

Department of Statistics, University of Toronto, M5S 3G3 Toronto, Ontario, Canada

e-mail: jeff@math.toronto.edu

\section{J. Segers}

Institut de statistique, Université catholique de Louvain, Voie du Roman Pays 20, 1348 Louvain-la-Neuve, Belgium

e-mail: johan.segers@uclouvain.be

\section{B. Sousa}

Departamento de Matemática para a Ciância e Tecnologia, Universidade do Minho, 4800-058 Guimarães, Portugal 


$$
\begin{aligned}
& \stackrel{\text { (B) }}{\leq} \int_{u}^{x_{+}} \frac{1}{V(u)}\left(\int_{u}^{x_{+}} V(y) \pi(\mathrm{d} y)+V(x) R \rho^{k}\right) \frac{\pi(\mathrm{d} x)}{\pi(u, \infty)} \\
& =\int_{u}^{x_{+}} \frac{V(y)}{V(u)} \pi(\mathrm{d} y)+R \rho^{k} \int_{u}^{x_{+}} \frac{V(x)}{V(u)} \frac{\pi(\mathrm{d} x)}{\pi(u, \infty)} \\
& =\left\{\operatorname{Pr}(X>U)+R \rho^{k}\right\} \mathrm{E}\left[\frac{V(X)}{V(u)} \mid X>u\right] .
\end{aligned}
$$

Explanation: (A) since $u \leq y$ and since $V$ is positive and non-decreasing; (B) by equation (2.5) in the paper, for certain constants $R>0$ and $0<\rho<1$. Now denote

$$
C=\limsup _{u \rightarrow \infty} \mathrm{E}\left[\frac{V(X)}{V(u)} \mid X>u\right] .
$$

Let $k$ be sufficiently large such that

$$
R \sum_{i=k}^{\infty} \rho^{i}<\frac{1}{2 C}
$$

The rest of the proof now is as in the paper.

Acknowledgement The authors wish to thank SANA LOUHICHI, Université de Paris-Sud, for having pointed out the error. 